\title{
Quantum-Based SI Traceable Electric-Field Probe
}

\author{
Joshua A. Gordon ${ }^{\# 1}$, Christopher L. Holloway ${ }^{\# 2}$, Steve Jefferts ${ }^{* 3}$, Tom Heavner ${ }^{* 4}$ \\ \#National Institute of Standards and Technology \\ \#Electromagnetics Division \\ 325 Broadway, Boulder, CO 80305, USA \\ jagordon@boulder.nist.gov, holloway@boulder.nist.gov \\ *National Institute of Standards and Technology \\ Time and Frequency Division \\ 325 Broadway, Boulder, CO 80305, USA \\ ${ }^{3}$ jefferts@boulder.nist.gov, ${ }^{4}$ heavner@boulder.nist.gov
}

\begin{abstract}
We are presently investigating the feasibility of developing a technique that will allow direct traceable microwave electric field ( $E$-field) measurements. The new approach is based on atomic rf-resonance spectroscopy, where an applied electrical field causes a transition between high-lying Rydberg states of an atom. The new technique will allow direct $E$-field measurements traceable to fundamental physical constants and SI units. If successful, this self calibrating probe will provide more accurate field measurements along with better sensitivity than do present techniques. Direct traceable $E$-field measurements with sensitivities below $0.1 \mathrm{~V} / \mathrm{m}$ could be possible.
\end{abstract}

\section{INTRODUCTION}

The proposed effort addresses the current lack of SI traceability in radio-frequency (RF) field measurements and the limitations this poses to areas of research requiring precise and accurate RF field measurements. E-field probe calibration is currently achieved by measuring a known reference field distribution that has been generated by a specially designed RF source. The field that is generated for calibration depends on the geometry of the RF source. Field measurements with current probes can at best only be traced to the geometrical parameters which prescribe the calibration reference field, and therefore RF probe measurements using current techniques lack direct traceability to SI units. The proposed quantumbased SI traceable electric-field probe will allow direct $E$-field measurements traceable to fundamental physical constants and SI units, and provide more accurate field measurements and better sensitivity than present techniques do. Direct SI traceable $E$-field measurements with sensitivities below $0.1 \mathrm{~V} / \mathrm{m}$ could be possible. Having a highly accurate and precise fiber-optic quantum-based SI-traceable E-field probe would extend the limits of high performance RF systems and could open up new areas of research in RF antenna design, RF

U.S. Government work not protected by U.S. copyright sensor array technology, and biomedical and nano-electronic systems measurement and design.

\section{CONCEPT}

The feasibility of developing a technique that will allow direct SI units linked to microwave electric field ( $E$-field) measurements will be investigated. Having NIST traceable microwave $E$-field measurement techniques is crucial to providing fundamental standards and traceability for critical quantities in electromagnetics. A probe based on atomic RFresonance spectroscopy will allow direct $E$-field measurements of both very weak and very strong fields over a large range of frequencies traceable to fundamental physical constants and SI units. Such a probe would provide more accurate field measurements, and a better sensitivity than present techniques. Direct traceable $E$-field measurements with sensitivities below $0.1 \mathrm{~V} / \mathrm{m}$ could be possible. This approach consists of optically exciting atoms to a Rydberg state and then applying an RF field. An applied electric field (E) can induce a transition of two states in Rydberg atoms (atoms in high energy states or with large quantum numbers). This transition can be detected by measuring the change in the resulting atomic fluorescence relative to the case when there is no applied field. This is illustrated in Figure 1, where the fluorescence at $819.7 \mathrm{~nm}$ would not be present in the absence of the applied $E$-field. It can be shown that this transition occurs when the product of the Rabi frequency (which is a function of the applied field strength, Planck's constant, and the atomic electric dipole matrix element) and the time that the field is applied are equal to $\pi[1]$, or

$$
E_{0}=\frac{h}{d \tau}
$$

where $h$ is Planck's constant, $E_{0}$ is the magnitude of the applied field (i.e., $\left.E=E_{0} \cos (\omega t)\right), \tau$ is the duration of 
the applied field, and $d$ is the atomic electric dipole matrix element. $d$ is a function of transition states of interest and is known to within $1 \%$. This approach consists of exciting atoms to a Rydberg state (by use of two lasers operating at different wavelengths) and then applying an electric field for time duration $\tau$. By observing the optical radiation with and without the applied field one determines when the desired transition has occurred. By use of (1), the measurement of the electric field is reduced to determining when a transition has occurred for a given $\tau$ and $d$. Similar experiments have been demonstrated for other applications [2,3], and it appears possible to apply this approach to the microwave $E$-field probe application. The RF frequencies where these resonances occur are a function of the atoms used. For example, for applied fields with frequencies of 1 to $10 \mathrm{GHz}$, lithium (Li) will be required, sodium ( $\mathrm{Na}$ ) can be used for 10 to $40 \mathrm{GHz}$, for 10 to $20 \mathrm{GHz}$ rubidium $(\mathrm{Rb})$ can be used, and for 100 to $500 \mathrm{GHz}$ cesium (Cs) is required. Measurements at only a few of these frequencies would allow a direct calibration of existing probes. This approach will be compared with $E$-field measurements from our existing probes. In this effort the capability of spectroscopy on Rydberg states of the sodium $\mathrm{Na}$ atom will be developed first. Figure 1 shows the energy levels and pumping scheme required for sodium.

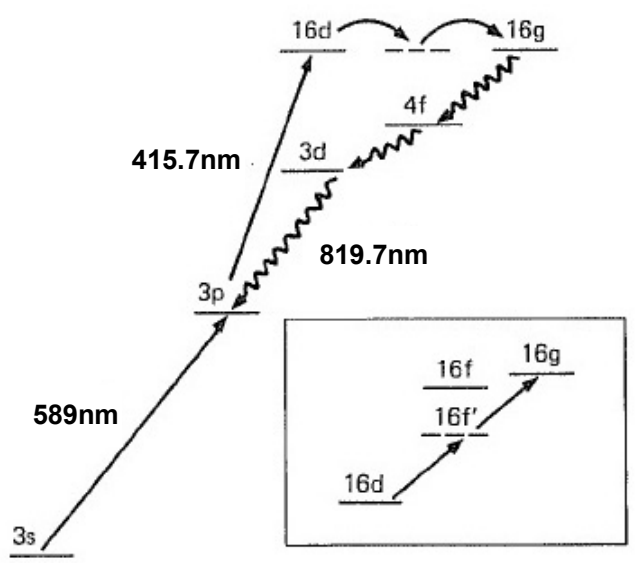

Figure 1: Relevant energy levels of sodium. (Taken From [1])

It is envisioned that the probe will consist of an extremely compact gas cell constructed from a hollow-core photonic crystal fiber (HC-PCF) that is filled with sodium atoms. This gas cell will be coupled to optical fibers that will be used to deliver the excitation laser light and to relay the resulting atomic fluorescence signal to a detector. This is shown in Figures 2 and 3 below.
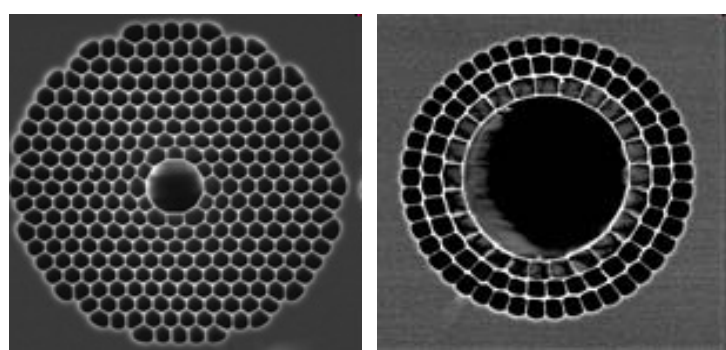

Hollow Core Photonic Band-Gap Fiber

Fig. 2 Hollow core photonic crystal fiber

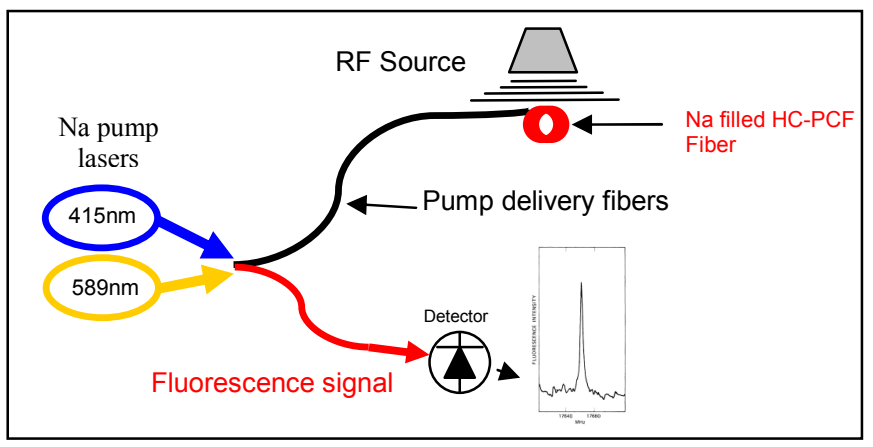

Fig. 3 Fiber based system schematic

\section{RF PROBE DESIGN INOVATION}

Several innovative ideas will be implemented in this effort. Use of RF spectroscopy allows for an exchange of energy from the $\mathrm{RF}$ regime to the optical regime via quantum mechanical mechanisms of Rydberg atoms. This in itself is an innovative approach to registering RF field strengths. Advantages to this approach are that there is no use of RF current generation to provide the measurement, unlike traditional RF probes that use metals and induce a current in an antenna. Therefore there is no risk of re-radiation or coupling RF fields from the probe back to the source. Such coupling can be a significant source of error in near-field type measurements. Innovation in the design of the probe using gas-filled hollow core photonic crystal fiber (HC-PCF) is proposed for this effort. Gas cells used in atomic physics experiments are typically made of a glass cylinder several centimeters in length and a few centimeters in diameter. It was identified early on that such a cell would be insufficient for use as an RF probe, due to the gross field perturbations that would result due to resonance inside the glass walls and within the volume of this type of cell at RF frequencies. This is shown in Figures 4 and 5. 

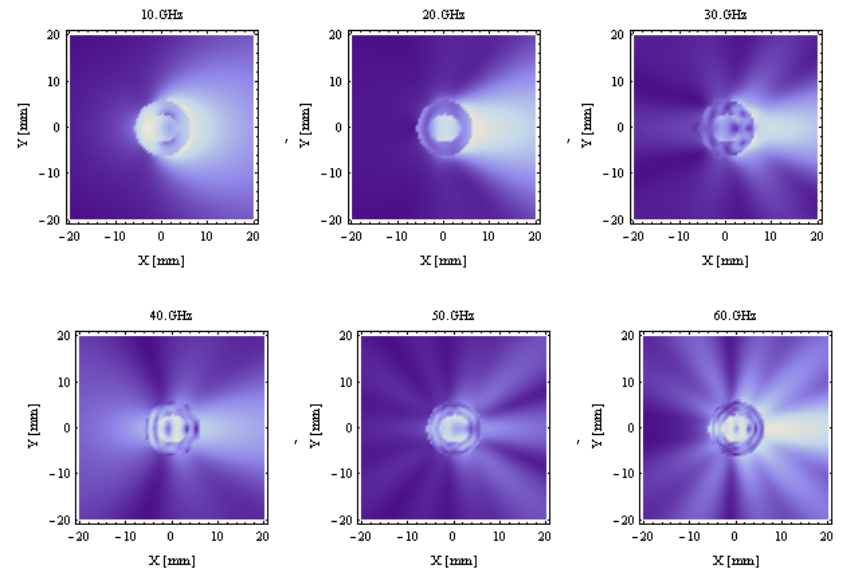

Fig. 4 Axial view of field perturbations due to glass vapor cell showing higher-order modes as the RF frequency is varied from $10 \mathrm{GHz}$ to $60 \mathrm{GHz}$.
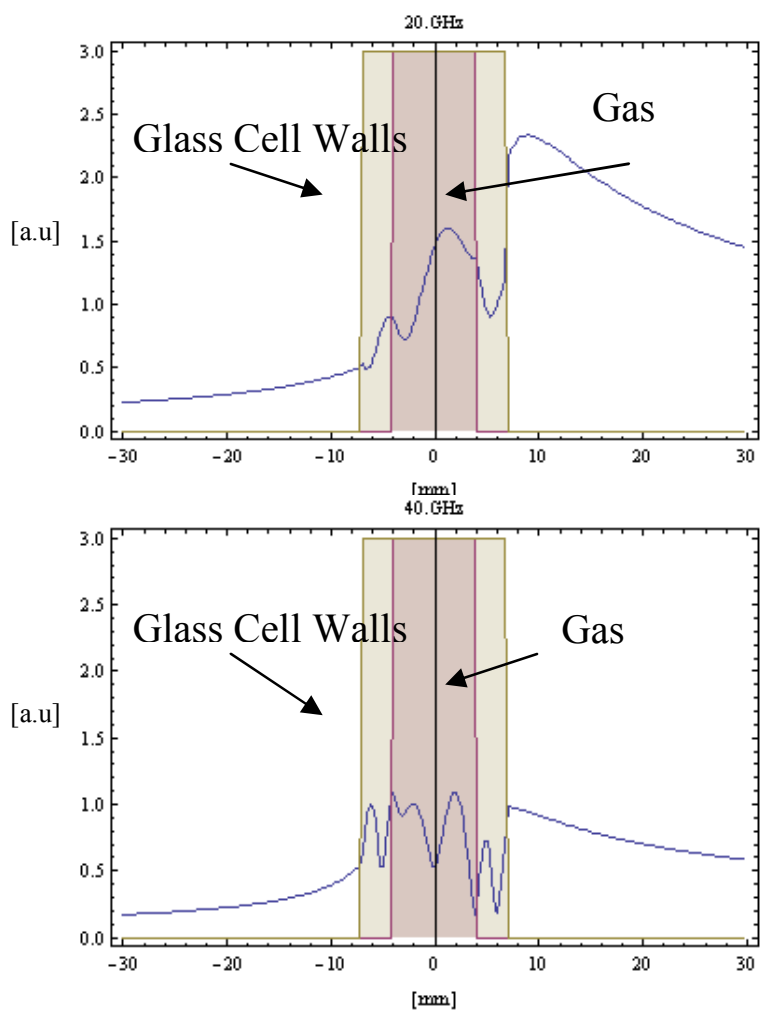

Fig. 5 Transverse view of field perturbations due to glass vapor cell for RF frequency of $20 \mathrm{GHz}$ to $40 \mathrm{GHz}$.

Use of HC-PCF filled with sodium gas is seen to be a solution to this problem. The dimensions of the HC-PCF are so small that the field perturbations are negligible within the core of the fiber. In addition, because HC-PCF is on the order of micrometers the probe can be made extremely small, allowing for point probe measurements. Other advantages of using HCPCF are that all of the laser sources can be relayed to the gas cell via fiber optics, and thus the probe is free to be placed in virtually any environment. With a typical cylindrical cell this would not be possible since the laser sources need to be steered into the volume of gas inside the cell, requiring auxiliary optics. Another very significant advantage of using a $\mathrm{HC}-\mathrm{PCF}$ is that nearly all of the photons from the fluorescence signal emanating from the gas of atoms are captured in the fiber and relayed to a detector. With a typical cylindrical gas cell, lenses must be used to capture the fluorescence photons from a very limited solid angle, with only the volume of atoms that are in the path of both the pump laser beams and the microwave field contributing to the fluorescence signal. Because the guiding mechanism in the HC-PCF is a band-gap effect the fiber can be bent around tight radii as small as a few millimeters. It is envisioned the HC-PCF could be wound up in a tight coil allowing interaction lengths of the microwave field and optical pump sources orders of magnitude longer than the few centimeters achievable with the cylindrical cell, thereby increasing the sensitivity of the probe by many orders of magnitude. In addition vapor levels of sodium within the fiber could be adjusted optically in real time via light induced atom desorption (LIAD) [4], as show in figure 6.
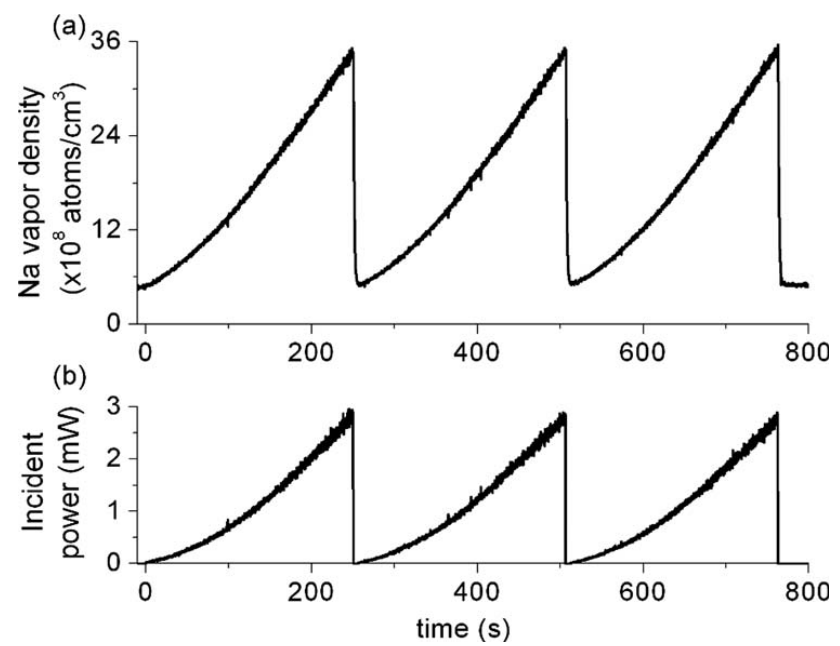

Fig. 6 LIAD of sodium showing atomic densities as a function of UV illumination power controllable in real time (see reference [4]).

Expanding on the compactness by use of HC-PCF, very compact laser sources will be developed. In this effort a VECSEL based $589 \mathrm{~nm}$ laser source will be developed by use of highly strained InGaAs-GaAs [5]. Until recently the 589 $\mathrm{nm}$ wavelength was only achievable by use of large dye lasers or bulky fiber lasers. It is envisioned that by use of semiconductor based lasers along with fiber optic technology, the fully developed quantum-based SI-traceable RF probe system can be miniaturized down to the size of a large cell phone or PDA. 


\section{POTENTIAL IMPACTS}

Better $E$-field measurement precision and lower uncertainties would allow the limits of high-performance systems to be extended, as well as improve the protection of persons exposed to electromagnetic fields through better field hazard probes. In addition, more precise and accurate electromagnetic field probes might open up defense and communication applications not now possible. For example, communication and sensing platforms are now densely populated with communications systems, radar, DE weapons, wireless control links, and other RF field based systems. Accuracy in $E$-field measurements is needed to optimize the compatibility of these systems. The use of fiber optics allows for probe dimensions to be on the order of micrometers in comparison to millimeters or centimeters for the wavelength of the fields being measured. Such small size could allow for highly accurate E-field measurements in packaged electronics of high density. Unlike previously developed micron-sized, fiberoptic based probes, which use electro-optic films for E-field detection [6], the use of atomic resonance in the proposed probe concept would provide self-calibrated measurements traceable to SI standard units. Because of this small size, the absence of metallic components in the probe design, and RFspectroscopy being an absorptive process, there would be little perturbation to the field being measured. Low field perturbations would result in virtually no coupling to the RF source due to scattering or re-radiation from the probe and therefore extremely low interference with the RF system being measured. Exploiting these properties and expanding the use of the single probe to multiple probes could open up new areas of measurement like the realization of imaging arrays useful for snap-shot mapping of microwave fields. Such snapshot mapping has potential applications for use in rapid measurement of radiation pattern and field uniformity, where conventional techniques require taking multiple point probe measurements and require long periods to map field patterns. The optical-fiber-based construction of the probe would open up possibilities of measuring RF fields in ways heretofore unachievable. The tip of the probe could be coated with metal aperture for integration of a near-field scanning bicroscope to the RF probe, allowing simultaneous near-field optical and RF measurements. In addition the fiber-based construction could also be integrated into endoscopic probes for medical applications, where it would be useful to investigate medically implanted electronic devices in vivo.

\section{CONCLUSION}

The feasibility of developing a technique that will allow direct traceable microwave electric field ( $E$-field) measurements is being explored. The new approach is based on atomic rfresonance spectroscopy, where an applied electrical field causes a transition between high-lying Rydberg states of an atom. The new technique will allow direct $E$-field measurements, traceable to fundamental physical constants and SI units. If successful, this self calibrating probe will provide more accurate field measurements and better sensitivity than present techniques, with direct traceable $E$ field measurements with sensitivities below $0.1 \mathrm{~V} / \mathrm{m}$ made possible. The new probe concept includes many innovations, including all-optical interrogation for measuring the RF field by use of a fiber-optic based designed as well as inherent self-calibration due to the use of atomic transitions for establishing field.

\section{ACKNOWLEDGEMENTS}

We acknowledge the support from Jim Bergquist, Michael Thorpe, Till Rosenband and Sara Bickman from the Physics Lab at NIST, Boulder CO for their generous help and use of equipment supporting this effort.

\section{REFERENCES}

[1] T.F. Gallagher, Rydberg Atoms, Cambridge University Press; N.Y., N.Y., 1994

[2] T. F. Gallagher, R. M. Hill, S. A. Edelstein, "Resonance measurements of $d-f$ splittings in highly excited states of sodium", Phys. Rev. A, vol. 13, no. 4, pp. 1448-1450, (1976)

[3] T. F. Gallagher, R. M. Hill, S. A. Edelstein, "Resonance measurements of $d$-f-g-h splittings in highly excited states of sodium", Phys. Rev. A, vol. 14, no. 2, pp. 744-50, (1976)

[4] A. Bogi, C. Marinelli, A. Burchianti, E. Mariotti, L. Moi, S. Gozzini, L. Marmugi, A. Lucchesini,"Full control of sodium vapor density in siloxane-coated cells using blue LED light-induced atomic desorption", Optics Lett. vol. 34, no. 17, 2643-2645 (2009)

[5] Mahmoud Fallahi, Li Fan, Yushi Kaneda, Chris Hessenius, Jörg Hader, Hongbo Li, Jerome V. Moloney, Bernardette Kunert, Wolfgang Stolz, Stephan W. Koch, James Murray, and Robert Bedford, "5-W Yellow Laser by Intracavity Frequency Doubling of High-Power VerticalExternal-Cavity Surface-Emitting Laser", IEEE Phot. Tech. Lett. vol. 20, no. 20, 1700-1702 (2008)

[6] http://www.nec.co.jp/press/en/0602/2101.html , "NEC Develops World's Smallest Fiber-Optic Electric Field Probe Utilizing Nanotechnology" (2006) 